SENSORY SYSTEMS

\section{Auditory advances}

Before hearing onset, the developing auditory system exhibits bursts of 'spontaneous' activity that are thought to contribute to circuit refinement; however, the specific influences of spontaneous activity on auditory system maturation are unclear. Findings reported in three new papers provide insight into the nature of spontaneous activity in the mouse auditory system and demonstrate its importance for the specification of cochlear neurons.

Both spontaneous and sensoryevoked auditory activity originate in the cochlea, where mechanosensory inner hair cells (IHCs) make synaptic contacts with bipolar spiral ganglion neurons (SGNs). The patterning of these connections and of the projections of SGNs to the brain means that the tonotopic segregation of auditory signals that begins in the cochlea is maintained throughout

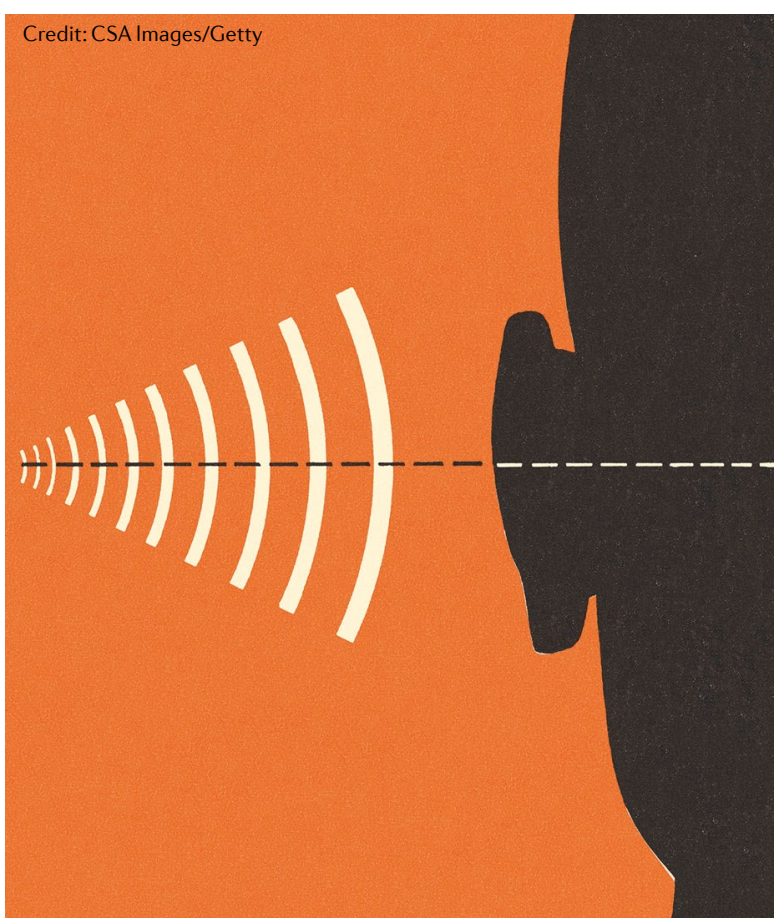

auditory processing pathways. In their new study, Babola et al. carried out in vivo imaging in awake mice to examine neuronal activity in the inferior colliculus (IC) and auditory cortex before the onset of hearing (which occurs $\sim 2$ weeks after birth). They found that populations of neurons destined to process similar sound frequencies exhibited correlated spontaneous activity during this critical developmental period both within and across auditory regions.

Babola et al. confirmed that this coordinated spontaneous activity relied upon spontaneous cochlear activity. However, they were surprised to find that mice lacking the vesicular glutamate transporter VGLUT3 (Vglut3-/- mice), which show disrupted IHC-SGN synaptic transmission, still exhibit spontaneous activity in SGNs and in the IC in the period before hearing onset. Further investigation identified a homeostatic plasticity mechanism in these mice, in which SGN sensitivity to potassium released by nearby supporting cells is enhanced, meaning that burst firing in these neurons continues in the absence of IHC synaptic input.

Cochlear SGNs were also the focus of the two complementary studies of Shrestha et al. and Sun et al. Previous studies have revealed SGN diversity in sound sensitivity and firing properties, which is thought to enable the detection of a wide dynamic range of sound signals. However, it was unknown whether these physiological distinctions map to specific molecular subtypes of SGN. Shrestha et al. and Sun et al. used single-cell RNA sequencing to examine the transcriptomic profiles of a large number of individual mouse SGNs, revealing that type I SGNs (which constitute $95 \%$ of all SGNs and are responsible for most sound transmission) can be divided into three subtypes that are characterized by their expression of specific marker proteins.

Next, the authors of these two studies considered the mechanisms by which these subtypes diversify during development. Using immunostaining and in situ hybridization to detect the molecular markers of each SGN subtype, they found that segregation of the subtypes begins around birth and is progressively refined in the first few postnatal weeks.

This suggested that spontaneous activity regulates SGN specification. Indeed, Shrestha et al. found that disruption of IHC-SGN signalling in Vglut $^{-/-}$mice impaired the consolidation of SGN subtype identities, and Sun et al. showed that mice lacking either VGLUT3 or a key component of the mechanotransduction complex present in IHCs exhibited distinct alterations in both SGN spontaneous activity and SGN specification, suggesting that the pattern of activity experienced by SGNs is important for their maturation.

These findings provide additional evidence for the important role of spontaneous activity in sensory system development. Such studies may lead to a better understanding of the molecular distinctions between different auditory neuron subtypes and of the plasticity that these cells exhibit in response to altered input. In turn, such advances may provide insight into the mechanisms driving congenital or acquired deafness.

Katherine Whalley

ORIGINAL ARTICLES Babola, T. A. et al. Homeostatic control of spontaneous activity in the developing auditory system. Neuron https://doi.org/10.1016/j.neuron.2018.07.004 (2018) | Shrestha, B. R. et al. Sensory neuron diversity in the inner ear is shaped by activity. Cell https://doi.org/10.1016/j.cell.2018.07.007 (2018) | Sun, S. et al. Hair cell mechanotransduction regulates spontaneous activity and spiral ganglion subtype specification in the auditory system. Cell https://doi.org/10.1016/j.cell.2018.07.008 (2018) 